\title{
Triggerable Multivalent Glyconanoparticles for Probing Carbohydrate-Carbohydrate Interactions
}

\author{
Sangho Won, ${ }^{\dagger}$ Steven Hindmarsh, ${ }^{\S}$ and Matthew I. Gibson, ${ }^{*}, \neq \oplus$ \\ ${ }^{\dagger}$ Department of Chemistry, University of Warwick, Gibbet Hill Road, Coventry CV4 7AL, U.K. \\ "Warwick Medical School, University of Warwick, Gibbet Hill Road, Coventry CV4 7AL, U.K. \\ ${ }^{\S}$ Department of Physics, University of Warwick, Gibbet Hill Road, Coventry CV4 7AL, U.K.
}

\section{Supporting Information}

ABSTRACT: Carbohydrate-carbohydrate interactions are proposed to be biologically significant but have lower affinities than the well-studied carbohydrate-protein interactions. Here we introduce multivalent glyconanostructures where the surface expression of lactose can be triggered by an external stimulus, and a gold nanoparticle core enables colorimetric signal outputs to probe binding. Macromolecular engineering of a responsive polymer "gate" enables the lactose moieties to be presented only when an external stimulus is present, mimicking how nature uses enzymes to dynamically regulate glycan expression. Two different carbohydrate-carbohydrate interactions are investigated using this tool.

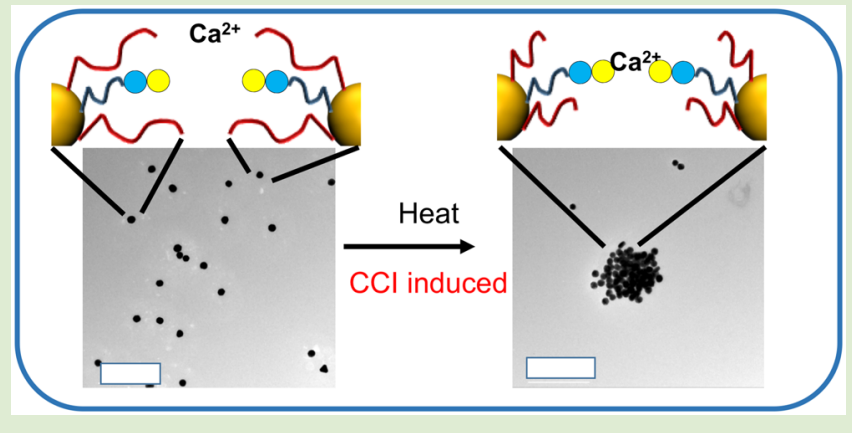

ells from across all kingdoms of life are coated with glycans (polysaccharides, glycolipids, glycoproteins) which provide internal and external cues for recognition, disease state, signal transduction, and more. ${ }^{1}$ The protein "readers" of glycans are termed lectins, which engage in relatively weak interactions with individual glycans with $\mathrm{mM}$ affinity. ${ }^{2,3}$ To overcome this, glycans are presented on cells in a multivalent format, enabling a nonlinear increase in binding affinity (often < nM), termed the "cluster glycoside effect", leading to dramatic increases in affinity. ${ }^{4}$ Inspired by this, synthetic materials bearing multiple carbohydrates have been developed which also have high affinity ${ }^{5}$ and can compete for native ligands in antiadhesion therapy ${ }^{6-8}$ or as biosensors. 9,10

While carbohydrate-lectin interactions have been extensively studied and a huge variety of tools exist to probe them, carbohydrate-carbohydrate interactions (CCIs) where the sugars directly engage with each other are much less studied, and their biological importance is not entirely clear. ${ }^{11}$ Glycosphingolipid CCIs have been implicated in the initial stages of cell-cell recognition, ${ }^{12}$ and the aggregation of marine sponges is linked to sulfated glycan CCIs. ${ }^{13,14}$ The affinity of CCIs is extremely weak, even compared to carbohydrate-lectin interactions $(\sim \mathrm{mM})$, and require divalent metal ions to bridge the glycans; multivalent presentation is essential to enable significant interaction forces to be generated. ${ }^{11,15,16}$ Murthy et al. have synthesized lactose-functional $\beta$-cyclodextrin which engages with GM3 glycolipid via a CCI to enable successful delivery of doxorubicin and may contribute to the lactose "trojan horse" effect for gaining entry into mammalian cells. ${ }^{17,18}$ Dendritic- and nanoparticle-based systems have been synthesized and evaluated for CCIs. ${ }^{11,16,19}$ These extremely weak interactions have been measured using solution $\mathrm{NMR}^{20}$ interfacial techniques such as Langmuir-Blodget ${ }^{19}$ and scanning probe microscopy, ${ }^{21}$ and also surface plasmon resonance (SPR). ${ }^{14}$ Seeberger and co-workers developed multivalent $4 \mathrm{~nm}$ silica glyconanoparticles to study GM3Gg3 interactions. ${ }^{16}$ Penades and co-workers have used glycanfunctional gold nanoparticles with SPR to demonstrate strong CCI multivalent effects. ${ }^{15}$ Russel and co-workers used PEGlactose functional gold particles for the plasmonic detection (red-blue color change) of CCIs, ${ }^{22}$ enabling simple and accessible read-outs, which have also found application in biosensing. ${ }^{10}$

A challenge in studying CCIs is that many of these are selfinteractions, e.g., lactose-lactose. Therefore, it is desirable to prevent spontaneous interactions between synthetic multivalent systems, by introducing an inducible "trigger" such that the glycan is presented with temporal or spacial control to underpin their study. In nature this is achieved by expression of glycosyltransferases which installs the glycan when required, depending on internal/external cues such as disease state. ${ }^{23,24}$ There are many examples of stimuli-responsive polymers, ${ }^{25}$ capable of changing their form (e.g., coli-globule transition) in response to redox, ${ }^{26}$ light, ${ }^{27}$ and metal ions. ${ }^{28}$ Mastrotto et al. have used the reversible collapse of poly $(N$-isopropylacrylamide) to present folate on particle surfaces for cellular delivery using thermal ${ }^{29}$ and $\mathrm{pH}$ stimuli. ${ }^{30}$ Sequential revealing of ligands on liposomes has enabled improved delivery of

Received: November 11, 2017

Accepted: January 16, 2018

Published: January 18, 2018 
liposome formulations. ${ }^{31}$ Spain and Alexander have used DNA strand displacement for oligonucleotide-specific ligand expression. ${ }^{32}$ Won et al. demonstrated that responsive polymers can provide a reversible steric "shield" to present glycans at gold nanoparticle surfaces, ensuring lectin binding only occurs when the system is activated. ${ }^{33}$

Herein we describe dynamic nanomaterials where the surface expression of glycans can be externally triggered, to induce a carbohydrate-carbohydrate interaction. We show that polymeric gates can effectively block a (shorter) polymer-bound glycan and present it at the surface of a gold nanoparticle on demand and exploit the coupling of gold particle SPRs to monitor the interaction colorimetrically under the control of a small temperature change. By engineering an inducible trigger, these systems will facilitate the study and exploitation of CCIs in biotechnological fields.

Our design principle requires a nonresponsive polymer bearing a glycan and a responsive polymer to act as the reversible gate: poly $(N$-hydroxyethyl acrylamide) (pHEA) and poly $(N$-isopropylacrylamide) (pNIPAM) were, respectively, chosen. ${ }^{33}$ pNIPAM was polymerized using CTA-2 (2(dodecylthiocarbonothioylthio)-2-methylpropionic acid). pHEA was polymerized using CTA-1 (pentafluorophenyl 2(dodecylthiocarbonothioylthio)-2-methylpropionic acid) which enables installation of amino-glycans onto the chain end by displacement of the PFP group (Figure 1). ${ }^{34}$ PFP-pHEA $_{15}$ was functionalized by addition of 2-amino-2-deoxy-D-galactose $\left(\mathrm{GalNH}_{2}\right)$ or 1-aminopropyl-D-lactose $\left(\mathrm{LacNH}_{2}\right)$, which was synthesized in 4 steps from lactose (SI). Addition of the glycans also results in cleavage of the RAFT agent, producing a terminal thiol suitable for immobilization onto citrate-stabilized gold nanoparticles. To ensure that the glycan-bearing polymer (pHEA) can be "hidden" by the responsive polymer (pNIPAM) the targeted degrees of polymerization were 15 and 50, respectively. ${ }^{33}$ SEC analysis showed slightly higher molecular weights than NMR for the pHEA and that polymers were well-defined, with $Đ<1.1 .{ }^{1} \mathrm{H}$ NMR and IR spectroscopies confirmed installation of the glycan. pNIPAM $_{50}$ had a cloud point of $38{ }^{\circ} \mathrm{C}$ at $1 \mathrm{mg} \mathrm{mL}^{-1}$ (Table 1). Once immobilized on the nanoparticle, the actual transition temperature will vary, ${ }^{35}$ and hence a full concentration-dependent study on this well-known polymer was not undertaken. ${ }^{26}$

$60 \mathrm{~nm}$ gold nanoparticles $\left(\mathrm{Au}_{60}\right)$ were coated with the indicated (Table 2) ratios of each polymer to generate particles bearing either pure glycan coatings or mixed glycan/responsive polymer coatings, which have been found to give grafting densities $\sim 0.3$ chains $/ \mathrm{nm}^{-2}$. ${ }^{36}$ Polymer coating was confirmed by the shift of the $S R_{\mathrm{MAX}}$ peak to longer wavelength, and dynamic light scattering showed an increase in diameter from 58 to $\sim 70 \mathrm{~nm}$. There was also a corresponding increase in the zeta potential. The particles had a net-negative charge as is widely reported for coating by this method, ${ }^{37}$ but the particles (see below) remain stable in buffered saline solutions. pNIPAM-containing polymers had cloud points above $50{ }^{\circ} \mathrm{C}$, but it is crucial to note that the actual chain collapse occurs below this temperature, which is essential for the present work. ${ }^{35}$

With this panel of nanoparticles bearing both responsive/ nonresponsive units and two different glycans in hand, control experiments were undertaken to demonstrate glycan-driven interactions at the particle surface. Lac-pHEA $15 @ \mathrm{Au}_{60}$ and GalNac-pHEA $15 @ \mathrm{Au}_{60}$ (i.e., nonresponsive coatings) were exposed to $0.1-100 \mathrm{mM}$ of $\mathrm{Ca}^{2+} \cdot \mathrm{Ca}^{2+}$ is the primary divalent

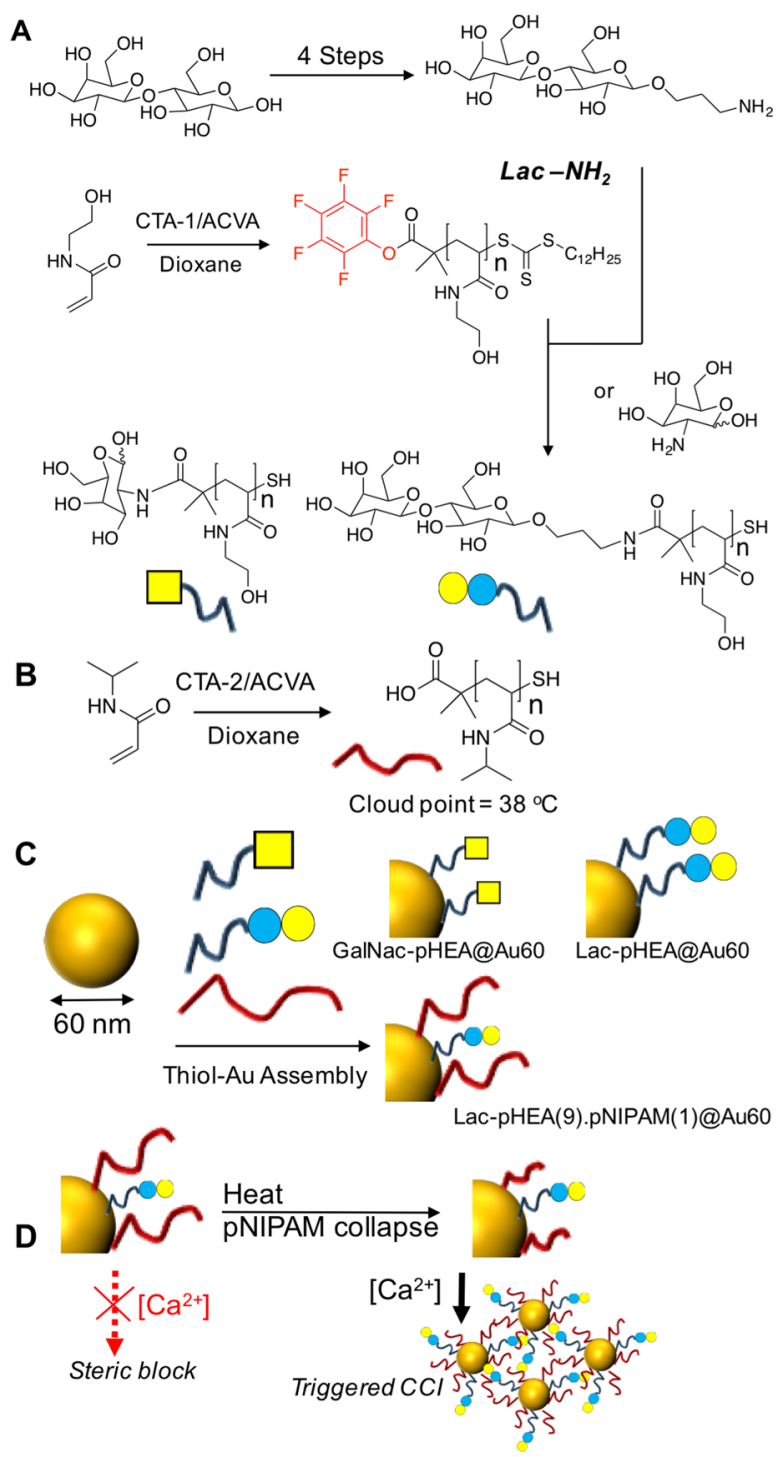

Figure 1. Synthesis of gated glycoparticles. (A) Synthesis of glycosylated pHEA; (B) synthesis of pNIPAM; (C) homo- and heterogeneous coating of gold nanoparticles; and (D) concept of yjr thermoresponsive polymer gate to control expression of lactose at the nanoparticle surface. Initiator $=4,4^{\prime}$-azobis (4-cyanopentanoic acid).

ion which bridges appropriate glycans to initiate the CCI. Following $30 \mathrm{~min}$ incubation Lac-pHEA $\mathrm{A}_{15} @ \mathrm{Au}_{60}$ showed distinct shifts in its UV-vis with the SPR $\mathrm{Abs}_{700}$ increasing, consistent with cross-linking (Figure 2). ${ }^{36,38}$ Conversely, addition of $\mathrm{Ca}^{2+}$ to GalNAc-pHEA $15 @ \mathrm{Au}_{60}$ showed very minor spectral changes with essentially zero aggregation (isotherms in SI). TEM analysis confirmed these observations (Figure 2E). GalNAc does not undergo self-self CCIs and hence excluded the possibility of nonspecific interactions due to changes in the ionic strength of the buffers. Similar results were obtained using $\mathrm{Mn}^{2+}$, also a divalent cation which can drive CCI (SI). To further corroborate the link between glycan presentation and aggregation, a lectin-mediated (carbohydrate-protein) interaction was also studied. SBA (soy bean agglutinin) was chosen as it has high affinity for GalNAc but lower affinity for lactose (i.e., opposite response to CCI expected). ${ }^{39}$ A serial dilution of SBA was added to both nanoparticles from 10 to $100 \mathrm{nM}$ and UV-vis spectra recorded (Figure 2C/D). SBA requires calcium to be present in the 
Table 1. Polymer Characterization

\begin{tabular}{|c|c|c|c|c|c|c|}
\hline polymer & {$[\mathrm{M}] /[\mathrm{CTA}] /[\mathrm{I}][\mathrm{mol}]$} & conv. $^{a}[\%]$ & $M_{\text {nTheo }}{ }^{b}\left[\mathrm{~g} \mathrm{~mol}^{-1}\right]$ & $M_{\mathrm{nSEC}}^{c}\left[\mathrm{~g} \mathrm{~mol}^{-1}\right]$ & $Ð[-]$ & $\mathrm{CP}^{d}\left[{ }^{\circ} \mathrm{C}\right]$ \\
\hline PFP-pHEA $_{15}$ & $15 / 1 / 0.2$ & $93 \%$ & 2100 & 4800 & 1.10 & - \\
\hline pNIPAM $_{50}$ & $50 / 1 / 0.2$ & $86 \%$ & 5200 & 7100 & 1.10 & 38 \\
\hline
\end{tabular}

Table 2. Nanoparticle Characterization

\begin{tabular}{lcccc}
\multicolumn{1}{c}{ particle } & $\mathrm{SPR}^{a}[\mathrm{~nm}]$ & $\operatorname{diameter}^{b}[\mathrm{~nm}]$ & $\mathrm{CP}^{c}\left[{ }^{\circ} \mathrm{C}\right]$ & - \\
$\mathrm{Au}_{60}$ & 534 & 58 & $\zeta^{d}[\mathrm{mV}]$ & $-41^{e}$ \\
pNIPAM $_{50} @ \mathrm{Au}_{60}$ & 538 & 75 & 59 & -15 \\
Lac-pHEA $_{15}(9) / \mathrm{pNIPAM}_{50}(1) @ \mathrm{Au}_{60}$ & 538 & 67 & 52 & -14 \\
Lac-pHEA $_{15} @ \mathrm{Au}_{60}$ & 537 & 66 & - & -11 \\
GalNAc-pHEA $_{15} @ \mathrm{Au}_{60}$ & 537 & 66 & - & -9
\end{tabular}

${ }^{a}$ Surface plasmon resonance band. ${ }^{b}$ Determined by DLS. ${ }^{c}$ Determined in water by UV-vis spectroscopy. ${ }^{d}$ Conducted in HEPES buffer unless noted. ${ }^{e}$ Conducted in water. Total gold particle concentration $\left(0.0255 \mathrm{mg} \mathrm{mL}^{-1}\right)$

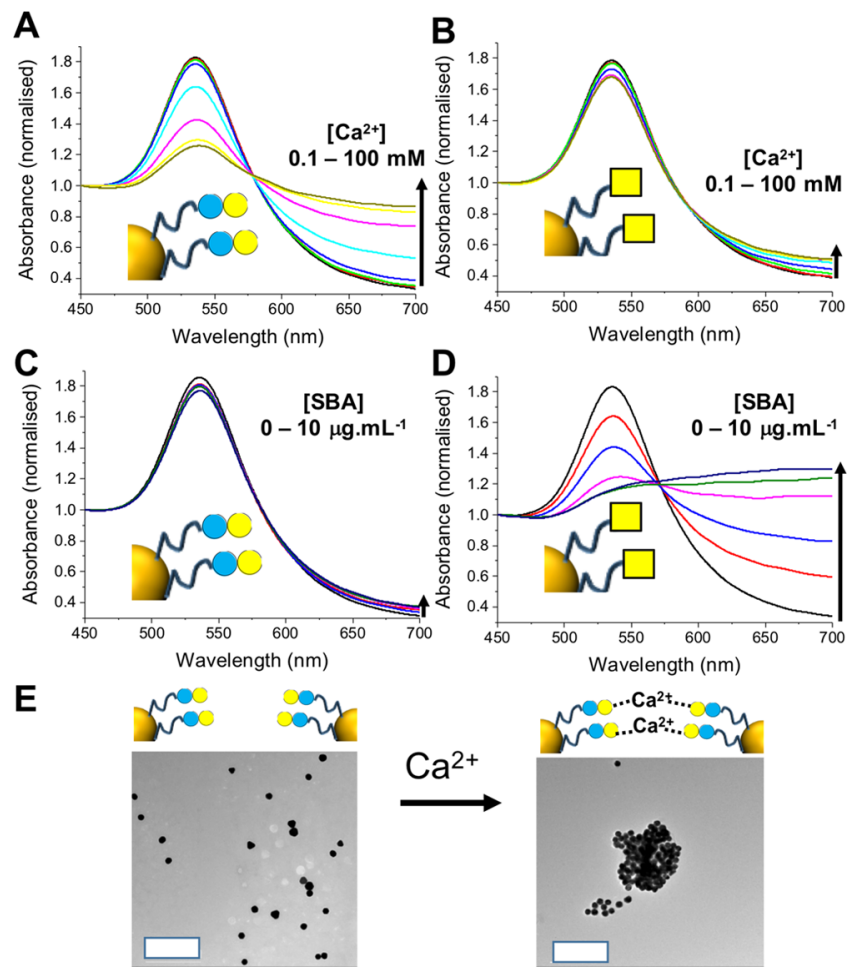

Figure 2. Calcium- and lectin-triggered aggregation of homogeneous glyconanoparticles. (A) Lac-pHEA 15 @Au $\mathrm{Au}_{60}$ with $\mathrm{Ca}^{2+}$; (B) GalNAcpHEA $15 @ A_{60}$ with SBA; (C) Lac-pHEA $15 @ A_{60}$ with SBA; (D) GalNAc-pHEA $15 @ \mathrm{Au}_{60}$ with SBA; and (E) TEM analysis of Lac$\mathrm{pHEA}_{15} @ \mathrm{Au}_{60}$ with and without $\mathrm{Ca}^{2+}$. Scale bar $=500 \mathrm{~nm}$.

binding mix, but at concentrations which did not cause CCI aggregation. Lac-pHEA ${ }_{15} @ \mathrm{Au}_{60}$ showed no aggregation, but GalNAc-pHEA ${ }_{15} @ \mathrm{Au}_{60}$ underwent strong dose-dependent binding to SBA. These control experiments confirmed the differential presentation of glycans on the nanoparticle surface that the end-group glycosylations provide sufficient density on the particle surface to induce a cluster-glycoside enhancement.

The above data demonstrated the challenge of self-self CCI study; when lactose-functional materials are placed in the biological buffers, spontaneous self-aggregation is always going to occur. This complicates detailed studies, especially in cellbased assays as the expression of lactose is static, not controllable, but has been reported to drive cell uptake by unknown mechanisms. ${ }^{17}$ To evaluate steric gating Lac-
$\mathrm{pHEA}_{15}: \mathrm{pNIPAM}_{50}$ ratio 9:1@Au $\mathrm{Au}_{60}$ was monitored for calcium-mediated aggregation at $20{ }^{\circ} \mathrm{C}$. At this temperature, the pNIPAM is fully extended and provides a steric block to CCIs, preventing aggregation even at $100 \mathrm{mM} \mathrm{Ca}^{2+}$ (Figure 3A). Time-dependent studies by monitoring $\mathrm{Abs}_{700}$ showed only a very slow change, and TEM revealed no evidence of aggregation (Figure 3C/D). Hence it can be seen that just 10 mol \% of pNIPAM on the particle surface can sterically block CCIs and is "gate closed".

Identical experiments as above were conducted but this time at $40{ }^{\circ} \mathrm{C}$ which is above the chain collapse temperature of pNIPAM but below the threshold for them to aggregate (avoiding false positives, see Table 2) (Figure 3E/F). Addition of $\mathrm{Ca}^{2+}$ leads to a clear increase in $\mathrm{Abs}_{700}$, and time-dependent monitoring of these showed rapid aggregation significantly faster and to a greater extent than that at $20{ }^{\circ} \mathrm{C}$. TEM confirmed the formation of aggregates in the presence of $\mathrm{Ca}^{2+}$ but that in the absence of $\mathrm{Ca}^{2+}$ (even at $40{ }^{\circ} \mathrm{C}$ ) there were no nonspecific particle-particle interactions. This is the first example of using an external trigger to control a CCI interaction and provides a dynamic tool for studying this area of glycoscience.

To further demonstrate the potential of these responsive nanoparticles, an assay was devised to mimic cell-surface hetero-CCI interactions, namely, lactose-GM-3. ${ }^{18}$ A polystyrene plate was modified by physiosorption of GM-3 glycolipid to create a cellular membrane mimic substrate. This was then incubated with both static and dynamic nanoparticles and binding evaluated by UV-vis and high-resolution scanning electron microscopy (SEM) after washing (Figure 4A). Due to the low concentration (a monolayer) low UV-vis signals were obtained, but the presence of the SPR peak at $\sim 550 \mathrm{~nm}$ could be seen when Lac-pHEA@ $@ \mathrm{Au}_{60}$ was incubated with calcium at $20{ }^{\circ} \mathrm{C}$; however, no SPR peak was seen for the other static particles (Figure 4B). Figure 4C shows the dynamic particles, $\operatorname{Lac} \mathrm{pHEA}_{15}(9) / \mathrm{pNIPAM}_{50}(1) @ \mathrm{Au}_{60}$, at both 20 and $40{ }^{\circ} \mathrm{C}$, showing nanoparticles were only bound at the higher temperature, when the gate is "open". SEM of the GM-3 surfaces corroborated the observations from UV-vis. Figure 4D/E shows that static nanoparticles are captured by GM-3 only for lactose not GalNAc functionality. Figure 4F shows the dynamic nanoparticles with "gate closed" at $20^{\circ} \mathrm{C}$ (no binding) and Figure $4 \mathrm{G}$ with "gate open" at $40{ }^{\circ} \mathrm{C}$ with significant LacGM-3 interactions occurring. 
A
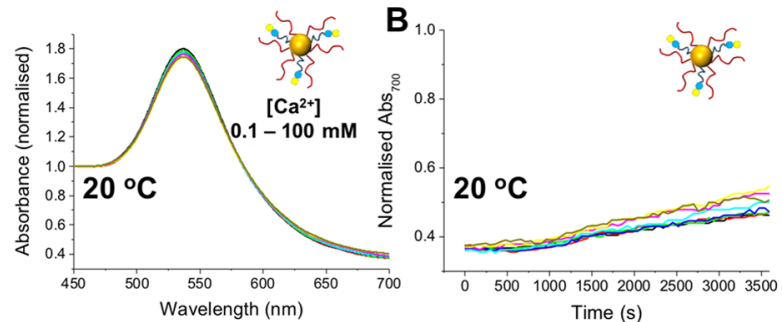

C

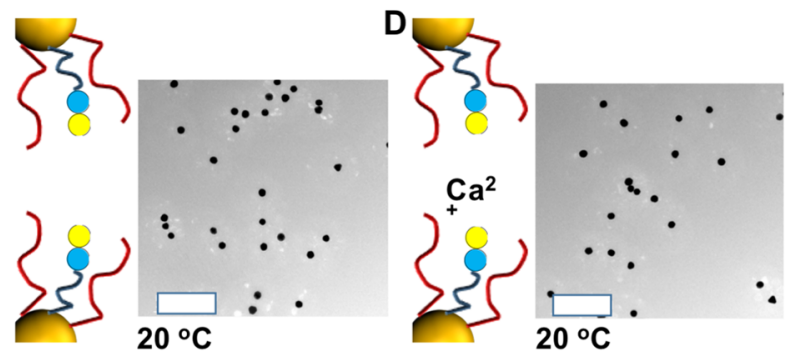

E

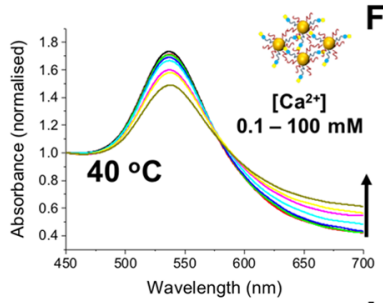

G

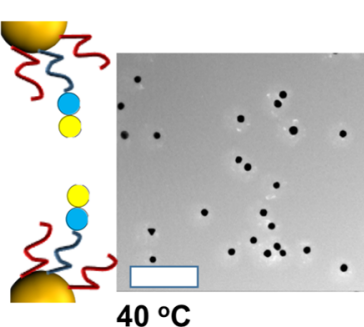

$\mathbf{F}$

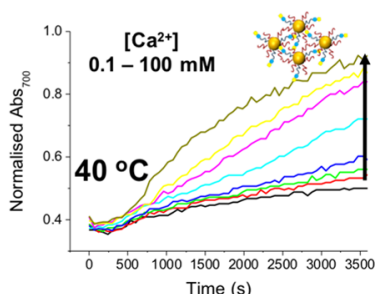

H

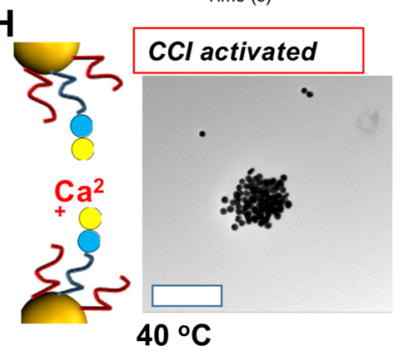

Figure 3. Temperature-controlled gating of lactose expression at a Lac-pHEA $15: \mathrm{pNIPAM}_{50}$ ratio of 9:1 $@ \mathrm{Au}_{60}$ surfaces. Ca ${ }^{2+}$ addition at $20{ }^{\circ} \mathrm{C}$ at (A) full UV-vis and (B) time dependence. TEM analysis (C) without and (D) with calcium showing no aggregation. $\mathrm{Ca}^{2+}$ addition at $40{ }^{\circ} \mathrm{C}$ at $(\mathrm{E})$ full $\mathrm{UV}$-vis and $(\mathrm{F})$ time dependence. TEM analysis $(\mathrm{G})$ without and $(\mathrm{H})$ with calcium showing selective $\mathrm{CCI}$ activation. Scale bar $=500 \mathrm{~nm}$.

Herein, we have demonstrated stimuli-responsive, "triggerable" multivalent glyconanoparticles for the study of carbohydrate-carbohydrate interactions. At the surface of gold nanoparticles nonresponsive polymers were used to anchor lactose and a responsive polymer, which can collapse upon application of a thermal stimulus used as a steric "gate". By exploiting this trigger, and the unique optical properties of the gold nanoparticle core, it was possible to monitor aggregation due to the lactose-lactose interactions in a label-free manner and upon application of the external stimulus. The hetero LacGM-3 interaction was also probed by this method using a binding assay which mimics a cell-surface interaction, demonstrating selective binding only upon application of the stimulus. This method enables temporal control of when the glycans are expressed on the nanoparticle surface, comparable to enzymatic control in nature preventing unwanted spontaneous interactions, entirely under the operator's control. This will facilitate the study and exploitation of multivalent carbohydrate interactions.

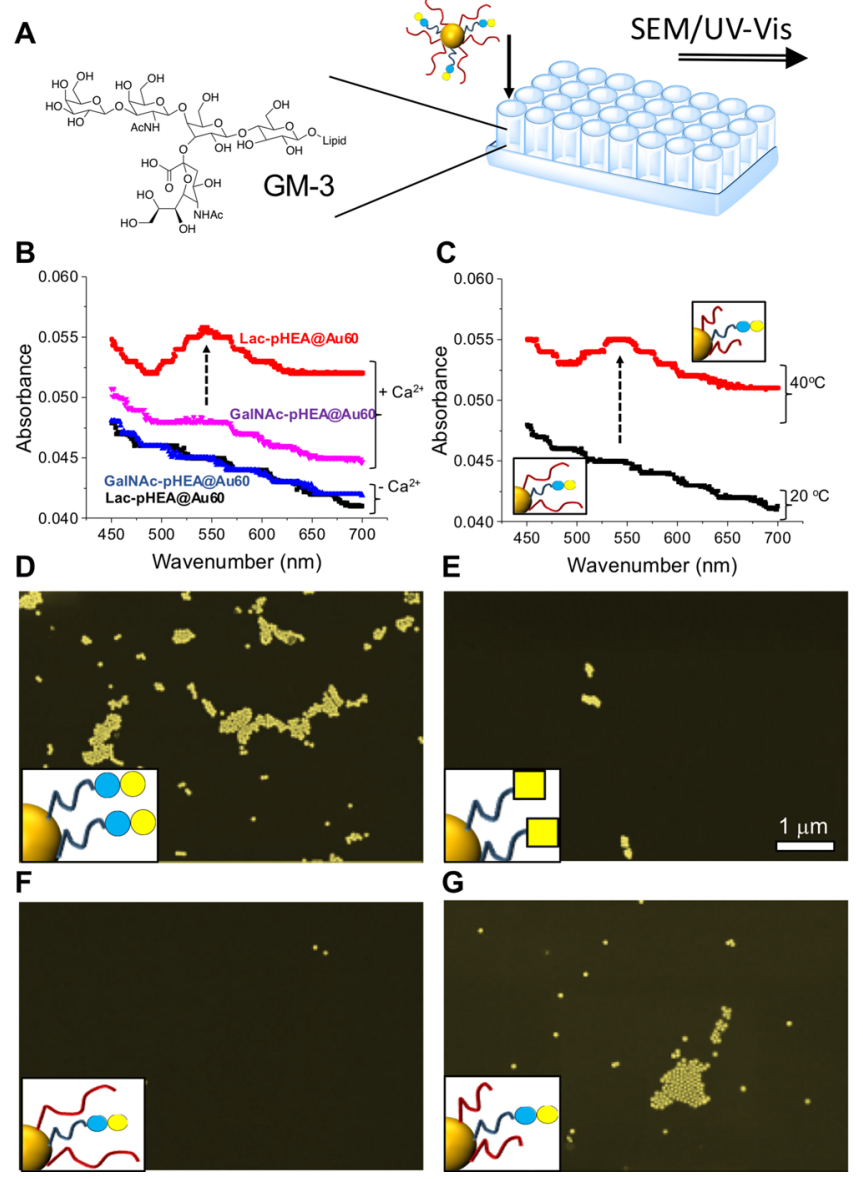

Figure 4. Interactions between glyconanoparticles and the GM-3 surface. (A) Experimental concept. (B) UV-vis analysis of the GM-3 surface after incubation with "static" nanoparticles at fixed temperature. (C) UV-Vis with dynamic nanoparticles $+\mathrm{Ca}^{2+}$. False-color SEM at GM-3 surfaces of (D) Lac-pHEA $15 @ \mathrm{Au}_{60}$ at $20{ }^{\circ} \mathrm{C}$; (E) GalNAc-pHEA $15 @ \mathrm{Au}_{60}$ at $20{ }^{\circ} \mathrm{C}$; (F) Lac-pHEA 15 (9)/pNIPAM p $_{50}(1)$ $@ \mathrm{Au}_{60}$ at $20{ }^{\circ} \mathrm{C}$; and (G) Lac-pHEA 15 (9)/pNIPAM ${ }_{50}(1) @ \mathrm{Au}_{60}$ at 40 ${ }^{\circ} \mathrm{C}$.

\section{ASSOCIATED CONTENT}

\section{S Supporting Information}

The Supporting Information is available free of charge on the ACS Publications website at DOI: 10.1021/acsmacrolett.7b00891.

Experimental procedures and characterization data plus additional binding experiments (PDF)

\section{AUTHOR INFORMATION}

\section{Corresponding Author}

*E-mail: m.i.gibson@warwick.ac.uk (M. I. Gibson).

\section{ORCID}

Matthew I. Gibson: 0000-0002-8297-1278

\section{Author Contributions}

The manuscript was written through contributions of all authors. All authors have given approval to the final version.

Notes

The authors declare no competing financial interest. 


\section{ACKNOWLEDGMENTS}

The University of Warwick is thanked for a Chancellors International Scholarship for SW. MIG holds an ERC starter grant (CRYOMAT 638661). The Electron Microscopy research technology platform and photoemission facility were supported by Advantage West Midlands and the ERDF.

\section{REFERENCES}

(1) Bertozzi, C. R.; Kiessling; L, L. Chemical Glycobiology. Science (Washington, DC, U. S.) 2001, 291 (5512), 2357-2364.

(2) Dam, T. K.; Brewer, C. F. Lectins as Pattern Recognition Molecules: The Effects of Epitope Density in Innate Immunity. Glycobiology 2010, 20 (3), 270-279.

(3) Ambrosi, M.; Cameron, N. R.; Davis, B. G. Lectins: Tools for the Molecular Understanding of the Glycocode. Org. Biomol. Chem. 2005, 3 (9), 1593-1608.

(4) Lundquist, J. J.; Toone, E. J. The Cluster Glycoside Effect. Chem. Rev. 2002, 102 (2), 555-578.

(5) Kiessling, L. L.; Grim, J. C. Glycopolymer Probes of Signal Transduction. Chem. Soc. Rev. 2013, 42 (10), 4476-4491.

(6) Kitov, P. I.; Sadowska, J. M.; Mulvey, G.; Armstrong, G. D.; Ling, H.; Pannu, N. S.; Read, R. J.; Bundle, D. R. Shiga-like Toxins Are Neutralized by Tailored Multivalent Carbohydrate Ligands. Nature 2000, 403 (6770), 669-672.

(7) Richards, S.-J.; Jones, M. W.; Hunaban, M.; Haddleton, D. M.; Gibson, M. I. Probing Bacterial-Toxin Inhibition with Synthetic Glycopolymers Prepared by Tandem Post-Polymerization Modification: Role of Linker Length and Carbohydrate Density. Angew. Chem., Int. Ed. 2012, 51 (31), 7812-7816.

(8) Becer, C. R.; Gibson, M. I.; Geng, J.; Ilyas, R.; Wallis, R.; Mitchell, D. A.; Haddleton, D. M. High-Affinity Glycopolymer Binding to Human DC-SIGN and Disruption of DC-SIGN Interactions with HIV Envelope Glycoprotein. J. Am. Chem. Soc. 2010, 132 (43), 1513015132.

(9) Otten, L.; Vlachou, D.; Richards, S.-J.; Gibson, M. I. Glycan Heterogeneity On Gold Nanoparticles Increases Lectin Discrimination Capacity in Label-Free Multiplexed Bioassays. Analyst 2016, 141 (14), $4305-4312$

(10) Marin, M. J.; Rashid, A.; Rejzek, M.; Fairhurst, S. A.; Wharton, S. A.; Martin, S. R.; McCauley, J. W.; Wileman, T.; Field, R. A.; Russell, D. A. Glyconanoparticles for the Plasmonic Detection and Discrimination between Human and Avian Influenza Virus. Org. Biomol. Chem. 2013, 11 (41), 7101-7107.

(11) de la Fuente, J. M.; Penadés, S. Understanding CarbohydrateCarbohydrate Interactions by Means of Glyconanotechnology. Glycoconjugate J. 2004, 21, 149-163.

(12) Bucior, I.; Scheuring, S.; Engel, A.; Burger, M. M. CarbohydrateCarbohydrate Interaction Provides Adhesion Force and Specificity for Cellular Recognition. J. Cell Biol. 2004, 165 (4), 529-537.

(13) Vilanova, E.; Santos, G. R. C.; Aquino, R. S.; Valle-Delgado, J. J.; Anselmetti, D.; Fernàndez-Busquets, X.; Mourão, P. A. S. Carbohydrate-Carbohydrate Interactions Mediated by Sulfate Esters and Calcium Provide the Cell Adhesion Required for the Emergence of Early Metazoans. J. Biol. Chem. 2016, 291 (18), 9425-9437.

(14) Haseley, S. R.; Vermeer, H. J.; Kamerling, J. P.; Vliegenthart, J. F. Carbohydrate Self-Recognition Mediates Marine Sponge Cellular Adhesion. Proc. Natl. Acad. Sci. U. S. A. 2001, 98 (16), 9419-9424.

(15) Hernáiz, M. J.; De La Fuente, J. M.; Barrientos, Á. G.; Penadés, S. A Model System Mimicking Glycosphingolipid Clusters to Quantify Carbohydrate Self-Interactions by Surface Plasmon Resonance. Angew. Chem., Int. Ed. 2002, 41 (9), 1554-1557.

(16) Lai, C.-H.; Hütter, J.; Hsu, C.-W.; Tanaka, H.; Varela-Aramburu, S.; De Cola, L.; Lepenies, B.; Seeberger, P. H. Analysis of Carbohydrate-Carbohydrate Interactions Using Sugar-Functionalized Silicon Nanoparticles for Cell Imaging. Nano Lett. 2016, 16 (1), 807811.

(17) Benito-Alifonso, D.; Tremel, S.; Hou, B.; Lockyear, H.; Mantell, J.; Fermin, D. J.; Verkade, P.; Berry, M.; Galan, M. C. Lactose as A "trojan Horse" for Quantum Dot Cell Transport. Angew. Chem., Int. Ed. 2014, 53 (3), 810-814.

(18) Murthy, R. V.; Bavireddi, H.; Gade, M.; Kikkeri, R. Exploiting the Lactose-GM3 Interaction for Drug Delivery. ChemMedChem 2015, 10 (5), 792-796.

(19) Seah, N.; Santacroce, P. V.; Basu, A. Probing the Lactose-GM3 Carbohydrate - Carbohydrate Interaction with Glycodendrimers. Org. Lett. 2009, 11 (3), 559-562.

(20) Santos, J. I.; de Souza, A. C.; Cañada, F. J.; Martín-Santamaría, S.; Kamerling, J. P.; Jiménez-Barbero, J. Assessing CarbohydrateCarbohydrate Interactions by NMR Spectroscopy: The Trisaccharide Epitope from the Marine Sponge Microciona Prolifera. ChemBioChem 2009, 10 (3), 511-519.

(21) Carvalho de Souza, A.; Vliegenthart, J. F. G.; Kamerling, J. P. Gold Nanoparticles Coated with a Pyruvated Trisaccharide Epitope of the Extracellular Proteoglycan of Microciona Prolifera as Potential Tools to Explore Carbohydrate-Mediated Cell Recognition. Org. Biomol. Chem. 2008, 6 (12), 2095.

(22) Reynolds, A. J.; Haines, A. H.; Russell, D. A. Gold Glyconanoparticles for Mimics and Measurement of Metal IonMediated Carbohydrate - Carbohydrate Interactions. Langmuir 2006, 22 (3), 1156-1163.

(23) Pinho, S. S.; Reis, C. A. Glycosylation in Cancer: Mechanisms and Clinical Implications. Nat. Rev. Cancer 2015, 15 (9), 540-555.

(24) Moremen, K. W.; Tiemeyer, M.; Nairn, A. V. Vertebrate Protein Glycosylation: Diversity, Synthesis and Function. Nat. Rev. Mol. Cell Biol. 2012, 13 (7), 448-462.

(25) Stuart, M. A. C.; Huck, W. T. S.; Genzer, J.; Müller, M.; Ober, C.; Stamm, M.; Sukhorukov, G. B.; Szleifer, I.; Tsukruk, V. V.; Urban, M.; Winnik, F.; Zauscher, S.; Luzinov, I.; Minko, S. Emerging Applications of Stimuli-Responsive Polymer Materials. Nat. Mater. 2010, 9 (2), 101-113.

(26) Phillips, D. J.; Gibson, M. I. Degradable Thermoresponsive Polymers Which Display Redox-Responsive LCST Behaviour. Chem. Commun. 2012, 48 (7), 1054-1056.

(27) Bertrand, O.; Gohy, J.-F. Photo-Responsive Polymers: Synthesis and Applications. Polym. Chem. 2016, 44 (1), 5539-5553.

(28) Phillips, D. J.; Prokes, I.; Davies, G.-L.; Gibson, M. I. Isothermally-Responsive Polymers Triggered by Selective Binding of Fe3+ to Siderophoric Catechol End-Groups. ACS Macro Lett. 2014, 3 (12), 1225-1229.

(29) Mastrotto, F.; Caliceti, P.; Amendola, V.; Bersani, S.; Magnusson, J. P.; Meneghetti, M.; Mantovani, G.; Alexander, C.; Salmaso, S. Polymer Control of Ligand Display on Gold Nanoparticles for Multimodal Switchable Cell Targeting. Chem. Commun. (Cambridge, U. K.) 2011, 47 (35), 9846-9848.

(30) Brazzale, C.; Mastrotto, F.; Moody, P.; Watson, P. D.; Balasso, A.; Malfanti, A.; Mantovani, G.; Caliceti, P.; Alexander, C.; Jones, A. T.; Salmaso, S. Control of Targeting Ligand Display by $\mathrm{pH}$-Responsive Polymers on Gold Nanoparticles Mediates Selective Entry into Cancer Cells. Nanoscale 2017, 9 (31), 11137-11147.

(31) Sawant, R. M.; Hurley, J. P.; Salmaso, S.; Kale, A.; Tolcheva, E.; Levchenko, T. S.; Torchilin, V. P. SMART” Drug Delivery Systems: Double-Targeted pH-Responsive Pharmaceutical Nanocarriers. Bioconjugate Chem. 2006, 17, 943.

(32) Magnusson, J. P.; Fernández-Trillo, F.; Sicilia, G.; Spain, S. G.; Alexander, C. Programmed Assembly of polymer-DNA Conjugate Nanoparticles with Optical Readout and Sequence-Specific Activation of Biorecognition. Nanoscale 2014, 6 (4), 2368-2374.

(33) Won, S.; Richards, S.-J.; Walker, M.; Gibson, M. I. Externally Controllable Glycan Presentation on Nanoparticle Surfaces to Modulate Lectin Recognition. Nanoscale Horiz. 2017, 2, 106-109.

(34) Richards, S.-J.; Gibson, M. I. Optimization of the Polymer Coating for Glycosylated Gold Nanoparticle Biosensors to Ensure Stability and Rapid Optical Readouts. ACS Macro Lett. 2014, 3 (10), 1004-1008

(35) Gibson, M. I.; O’Reilly, R. K. To Aggregate, or Not to Aggregate? Considerations in the Design and Application of Polymeric 
Thermally-Responsive Nanoparticles. Chem. Soc. Rev. 2013, 42 (17), 7204-7213.

(36) Richards, S.-J.; Otten, L.; Gibson, M. I. Glycosylated Gold Nanoparticle Libraries for Label-Free Multiplexed Lectin Biosensing. J. Mater. Chem. B 2016, 4 (18), 3046-3053.

(37) Zhang, Z.; Maji, S.; Antunes, A. B.; da, F.; De Rycke, R.; Zhang, Q.; Hoogenboom, R.; De Geest, B. G. Salt Plays a Pivotal Role in the Temperature-Responsive Aggregation and Layer-by-Layer Assembly of Polymer-Decorated Gold Nanoparticles. Chem. Mater. 2013, 25 (21), 4297-4303.

(38) Elghanian, R.; Storhoff, J. J.; Mucic, R. C.; Letsinger, R. L.; Mirkin, C. A. Selective Colorimetric Detection of Polynucleotides Based on the Distance-Dependent Optical Properties of Gold Nanoparticles. Science (Washington, DC, U. S.) 1997, 277 (5329), 1078-1081.

(39) Wu, A. M.; Lisowska, E.; Duk, M.; Yang, Z. Lectins as Tools in Glycoconjugate Research. Glycoconjugate Journal; Springer US; November 27, 2009; pp 899-913. 